\title{
Cost and contribution margin of transcatheter versus surgical aortic valve replacement
}

Fenton H. McCarthy, MD, MS, ${ }^{\mathrm{a}, \mathrm{b}}$ Danielle C. Savino, BS, ${ }^{\mathrm{b}}$ Chase R. Brown, MD, ${ }^{\mathrm{b}}$ Joseph E. Bavaria, MD, ${ }^{\mathrm{b}}$ Vinay Kini, MD, MS, ${ }^{a, c}$ Danielle D. Spragan, MD, ${ }^{b}$ Taylor R. Dibble, BS, ${ }^{b}$ Howard C. Herrmann, MD, ${ }^{c}$ Saif Anwaruddin, MD, ${ }^{\mathrm{c}}$ Jay Giri, MD, ${ }^{\mathrm{c}}$ Wilson Y. Szeto, MD, ${ }^{\mathrm{b}}$ Peter W. Groeneveld, MD, MS,,${ }^{\mathrm{a}, \mathrm{e}}$ and Nimesh D. Desai, MD, PhD ${ }^{\mathrm{a}, \mathrm{b}}$

\section{ABSTRACT}

Objective: To compare the cost of and payments for transcatheter aortic valve replacement (TAVR), a novel and expensive technology, and surgical aortic valve replacement (SAVR).

Methods: Medicare claims provided hospital charges, payments, and outcomes between January and December 2012. Hospital costs and charges were estimated using hospital-specific cost-to-charge ratios. Costs and payments were examined in propensity score- matched TAVR and SAVR patients.

Results: Medicare spent $\$ 215,770,200$ nationally on 4083 patients who underwent TAVR in 2012. Hospital costs were higher for TAVR patients (median, \$50,200; interquartile range [IQR], \$39,800-\$64,300) than for propensity-matched SAVR patients $(\$ 45,500 ; \mathrm{IQR}, \$ 34,500-\$ 63,300 ; P<.01)$, owing largely to higher estimated medical supply costs, including the implanted valve prosthesis. Postprocedure hospital length of stay (LOS) length was shorter for TAVR patients (median, 5 days [IQR, 4-8 days] vs 7 days [IQR, 5-9 days]; $P<.01$ ), as was total intensive care unit (ICU) LOS (median, 2 days [IQR, $0-5$ days] vs 3 days [IQR, 1-6 days]; $P<.01)$. Medicare payments were lower for TAVR hospitalizations (median, $\$ 49,500$; IQR, $\$ 36,900-\$ 64,600$ ) than for SAVR (median, \$50,400; IQR, \$37,400-\$65,800; $P<.01$ ). The median of the differences between payments and costs (contribution margin) was $-\$ 3380$ for TAVR hospitalizations and $\$ 2390$ for SAVR hospitalizations $(P<.01)$.

Conclusions: TAVR accounted for \$215 million in Medicare payments in its first year of clinical use. Among SAVR Medicare patients at a similar risk level, TAVR was associated with higher hospital costs despite shorter ICU LOS and hospital LOS. Overall and/or medical device cost reductions are needed for TAVR to have a net neutral financial impact on hospitals. (J Thorac Cardiovasc Surg 2017;154:1872-80)

The recent development of transcatheter aortic valve replacement (TAVR) presents a new therapeutic option for patients with severe aortic stenosis considered at least

\footnotetext{
From the ${ }^{\mathrm{a}}$ Leonard Davis Institute of Health Economics, Divisions of ${ }^{\mathrm{b}}$ Cardiovascular Surgery, and ${ }^{\mathrm{c} C}$ ardiology, and ${ }^{\mathrm{d}}$ Department of Medicine, University of Pennsylvania; and ${ }^{e}$ Philadelphia Veterans Affairs Medical Center's Center for Health Equity Research and Promotion, Philadelphia, Pa.

Received for publication March 8, 2016; revisions received May 28, 2017; accepted for publication June 8, 2017; available ahead of print July 13, 2017.

Address for reprints: Fenton H. McCarthy, MD, MS, Division of Cardiovascular Surgery, Hospital of the University of Pennsylvania, 3400 Spruce St, 6th Floor Silverstein, Philadelphia, PA (E-mail: Fenton.McCarthy@uphs.upenn.edu). 0022-5223/\$36.00

Copyright (c) 2017 by The American Association for Thoracic Surgery http://dx.doi.org/10.1016/j.jtcvs.2017.06.020
}

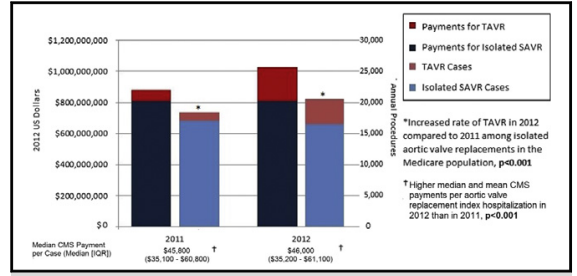

Annual CMS payments for TAVR and isolated SAVR in 2011 and 2012.

\section{Central Message}

Among patients with similar risks, TAVR is associated with higher hospital costs in comparison to SAVR despite shorter ICU and hospital stays.

\section{Perspective}

The financial impact of TAVR in the United States remains relatively unknown, and a rigorous economic comparison to the alternative therapy of SAVR is warranted.

See Editorial Commentary page 1881. 

Abbreviations and Acronyms
AVR $=$ aortic valve replacement
CMS $=$ Center for Medicare and Medicaid Services
DRG = diagnosis-related group
FDA $\quad=$ Food and Drug Administration
ICU = intensive care unit
LOS $\quad=$ length of stay
MEDPAR $=$ Medicare Provider Analysis and Review
SAVR = surgical aortic valve replacement
TAVR $=$ transcatheter aortic valve replacement

Administration (FDA) in November 2011, and by the end of 2012, more than 200 hospitals in the United States offered the procedure. ${ }^{4,5}$

Studies examining the relative cost-effectiveness of TAVR compared with standard medical therapy and/or SAVR have reported conflicting results using various cost metrics. An analysis of estimated expenditures for patients enrolled in early US clinical trials postulated that TAVR would be a cost-effective alternative to SAVR for patients considered at high operative risk and found no significant differences between TAVR and SAVR in hospital costs for the index admission or in 1-year cumulative costs. ${ }^{6}$ Compared with medical management in inoperable patients, results with TAVR have varied, demonstrating a favorable incremental cost-effectiveness ratio of $\$ 32,170$ per quality-adjusted life-year gained in Canada, but a less favorable incremental cost-effectiveness ratio of $\$ 116,500$ per quality-adjusted life-year gained in the United States. ${ }^{7,8}$ Compared with SAVR, TAVR performed outside the United States has variously been reported as significantly less costeffective, more cost-effective, and equally cost-effective. ${ }^{9-11}$ In addition, the results and conclusions from systematic reviews of the relative cost-effectiveness have been mixed. ${ }^{9,12,13}$ There is a paucity of studies examining the relationships among hospital costs, Medicare payments and total revenue associated with TAVR versus SAVR.

More specifically, little attention has been paid to the practical economic considerations impacting US hospitals offering TAVR, specifically hospital revenue, as well as the overall impact of the procedure on the US Medicare program at large. Because candidates for aortic valve replacement, and especially for TAVR, generally compose an elderly population, reimbursements to hospitals for the procedure and associated hospitalizations are obtained almost universally through Medicare. The use of TAVR in previously inoperable SAVR patients, the aging population, and the potential expansion of TAVR into lower-risk cohorts could significantly, if not exponentially, increase its use. As a result, TAVR represents a potentially growing source of expenditures for the Medicare program. Little is known regarding the relative cost of TAVR compared with SAVR - a comparable therapy in some patients. ${ }^{14}$ Our present analysis had 3 primary aims: (1) to describe US Medicare payments to hospitals associated with TAVR; (2) to compare the Medicare expenditures, estimated hospital costs, and net hospital contribution margin between TAVR and SAVR within a propensity score-matched sample; and (3) to identify the specific factors with the greatest influence on hospital costs and Medicare payments during TAVR or SAVR hospitalizations in the United States.

\section{METHODS}

\section{Study Sample and Medicare Data}

The study includes all fee-for-service Medicare beneficiaries. Patients and comorbidities were identified using Medicare's Medicare Provider Analysis and Review (MEDPAR) (for inpatient claims) and Carrier (for specific physician/supplier claims) files. Procedural utilization and billing data from the Center for Medicare and Medicaid Services (CMS) were used to identify all aortic valve replacement (AVR; SAVR and TAVR) hospitalizations occurring between January 1, 2012, and December 31, 2012. Cost and utilization data for all AVRs from 2011 is used for reference and represents an era primarily of clinical trial use of TAVR that preceded FDA approval. Using Health Care Procedure Classification System codes present on carrier claims collected by the CMS, 4083 TAVR and 16,530 SAVR index hospitalizations among Medicare fee-for-service patients were identified. The cost and number of isolated aortic valve replacement cases in 2012 was compared with claims in 2011 using adjusted 2012 US dollars from the US Bureau of Labor Statistics. ${ }^{15}$ Hospitalization, outpatient facility, and physician claims from these patients were linked by the Medicare beneficiary's patient identifier to obtain demographic information, diagnosis codes, procedure codes, admit and discharge dates, and financial data associated with each patient's index AVR hospitalization. Diagnosis codes present on admission were used to identify common comorbidities using a modified Elixhauser comorbidity method. ${ }^{16}$ Patients undergoing concomitant cardiac surgical procedures were also identified using procedure codes on carrier claims and were excluded.

\section{Cost and Payment Data}

Using Medicare data to answer questions regarding costs of TAVR and SAVR has unique advantages, because it includes all charges and payments from fee-for-service Medicare beneficiaries. For this study, actual hospital charges and insurance payments for all isolated AVR hospitalizations during calendar year 2012 were used to examine the financial impact of AVR in the Medicare population during the first year after FDA approval of TAVR. Hospital charges and Medicare payments were collected from Medicare's MEDPAR files, which represent final action (ie, completely adjudicated) claims on individual patient hospitalizations. This payment also accounts for the post-acute care payment adjustment-sometimes referred to as a "transfer penalty" - which can potentially result in a diagnosis-related group (DRG)-specific down-weighting of reimbursement for admissions that are shorter relative to the national mean length of stay (LOS). Hospital charges, which are subdivided into 30 hospital cost centers on MEDPAR claims, indicate each hospital departmental (eg, radiology), resource (eg, blood bank), and accommodation (eg, ward) charge for care provided during the index TAVR or SAVR admission. Payments, which are separately reported on MEDPAR claims as patient payments, private insurance payments, and Medicare payments, were also abstracted.

Hospital charges were converted to cost of care using hospital-specific cost-to-charge ratios from the 2015 Inpatient Prospective Payment System 
TABLE 1. Baseline characteristics of isolated SAVR and TAVR patients

\begin{tabular}{|c|c|c|c|c|}
\hline Characteristic & $\begin{array}{c}\text { All } \\
(n=20,613)\end{array}$ & $\begin{array}{c}\text { SAVR } \\
(\mathrm{n}=\mathbf{1 6 , 5 3 0 )}\end{array}$ & $\begin{array}{c}\text { TAVR } \\
(n=4083)\end{array}$ & $P$ value \\
\hline Age, $y$, mean \pm SD & $77 \pm 9.2$ & $75 \pm 8.8$ & $83 \pm 8.2$ & $<.001$ \\
\hline Male sex & $10,955(53)$ & $8847(54)$ & $2108(52)$ & .031 \\
\hline White race & $18,991(92)$ & $15,146(92)$ & $3845(94)$ & $<.001$ \\
\hline Black race & $892(4)$ & $751(5)$ & $141(3)$ & .002 \\
\hline Congestive heart failure & $12,191(59)$ & $8534(52)$ & $3657(90)$ & $<.001$ \\
\hline Pulmonary circulation disorder & $6133(30)$ & $4266(26)$ & $1867(46)$ & $<.001$ \\
\hline Peripheral vascular disease & $10,950(53)$ & $7877(48)$ & $3073(75)$ & $<.001$ \\
\hline Neurologic disorder & $2278(11)$ & $1649(10)$ & $629(15)$ & $<.001$ \\
\hline Chronic obstructive pulmonary disease & $7905(38)$ & $5727(35)$ & $2178(53)$ & $<.001$ \\
\hline Diabetes mellitus & $2943(14)$ & $2173(13)$ & $770(19)$ & $<.001$ \\
\hline Renal failure & $6053(29)$ & $4024(24)$ & $2029(50)$ & $<.001$ \\
\hline Liver disease & $631(3)$ & $466(3)$ & $165(4)$ & $<.001$ \\
\hline Coagulopathy & $3513(17)$ & $2708(16)$ & $805(20)$ & $<.001$ \\
\hline Obesity & $4687(23)$ & 3937 (24) & $750(18)$ & $<.001$ \\
\hline Weight loss & $1252(6)$ & $878(5)$ & $374(9)$ & $<.001$ \\
\hline Anemia & $7325(36)$ & $5150(31)$ & $2175(53)$ & $<.001$ \\
\hline Depression & $2588(13)$ & $1961(12)$ & $627(15)$ & $<.001$ \\
\hline Hypertension (complicated) & $19,004(92)$ & $15,090(91)$ & $3914(96)$ & $<.001$ \\
\hline
\end{tabular}

Data are $\mathrm{n}(\%)$ unless noted otherwise. SAVR, Surgical aortic valve replacement; TAVR, transcatheter aortic valve replacement; $S D$, standard deviation.

Impact File, which represents cost-to-charge ratios calculated using 2012 CMS cost data. Thus, cost of care was used as a proxy to estimate a hospital's total expenditures for each TAVR or SAVR hospitalization. The use of these data in Medicare cost analyses has been validated previously. ${ }^{17,18}$ Institutional contribution margins were calculated by taking the difference between the median cost and the payment for all patients at each institution along with the interquartile range. Physician fees were not included in the cost analysis, because this usually represents $<10 \%$ of hospital reimbursement. The perspective of this health economic analysis is costs and payments associated with individual patients in propensity score-matched TAVR and SAVR cohorts. Consideration of the impact of these costs and payments also includes institutional and societal (all Medicare fee-for-service patient) levels.

\section{Statistical Analysis and Propensity Matching}

Categorical variables were analyzed using Fisher's exact test, and continuous variables were analyzed using the 2 -sample $t$ test. In the propensity score-matched analyses, the paired $t$ test and McNemar's test were used for continuous and categorical variables, respectively. The Mann-Whitney nonparametric $U$ test was used for analyses of charges and payments due to a lack of normal distribution. A $P$ value $<.05$ was considered statistically significant.

Because of the significant differences in age, race, and incidence of several clinically significant comorbidities between TAVR and SAVR patients in the isolated AVR sample, a propensity score-matched sample was created to facilitate comparison among patients most likely to have similar risk and likelihood of undergoing either SAVR or TAVR. Propensity score-matched analysis was used to generate cohorts of TAVR and SAVR. The variables used to propensity score match TAVR and SAVR patients included male sex, age, black race, congestive heart failure, peripheral vascular disease, anemia, chronic obstructive pulmonary disease, pulmonary hypertension, renal failure, diabetes, neurologic disorders, obesity, depression, weight loss, and liver disease. A logistic regression that included demographic and risk factor variables was used to generate propensity scores for undergoing TAVR or SAVR for each patient in the sample. A total of 3304 1-to-1 pairs of TAVR and SAVR patients were identified using a greedy $5 \rightarrow 1$ digit match algorithm with nearest available pair matching. ${ }^{19}$ The c-statistic for the model was 0.834 . Three sensitivity analyses were performed: (1) multivariable logistic regression models with generalized estimating equations using an exchangeable correlation structure to assess risk factors for 30-day mortality; (2) limiting SAVR patients to only those from institutions that perform TAVR; and (3) comparing only transfemoral (TF) TAVR with SAVR. Data management, propensity score matching, and analyses were done using SAS version 9.4 (SAS Institute, Cary, NC).

\section{RESULTS}

\section{All TAVR and SAVR Patients}

After exclusions, 4083 TAVR patients and 16,530 SAVR patients undergoing isolated AVR in 2012 were identified. TAVR patients were older on average than the SAVR patients (mean, $83 \pm 8.2$ years vs $75 \pm 8.8$ years; $P<.01$ ), more likely to be white, and more likely to present with common comorbidities, including congestive heart failure, chronic pulmonary disease, diabetes, renal failure, and anemia (Table 1). Thirty-day mortality was also higher in TAVR patients than in SAVR patients in the entire sample $(6 \%$ vs $4 \% ; P<.01)$ (Table 2$)$. Postprocedure LOS was shorter following TAVR than following SAVR (median, 5 days [IQR, 4-8 days] vs 7 days [IQR, 5-9 days]; $P<.01$ ), as was total intensive care unit (ICU) LOS (median, 2 days [IQR, 0-5] vs 3 days [IQR, 1-6 days]; $P<.01)$. 
TABLE 2. Postoperative outcomes of all SAVR and TAVR patients versus propensity score-matched SAVR and TAVR patients

\begin{tabular}{|c|c|c|c|c|}
\hline$\frac{\text { Outcomes }}{\text { All SAVR and TAVR patients }}$ & $\frac{\text { All }}{\mathrm{n}=\mathbf{2 0 , 6 1 3}}$ & $\frac{\text { SAVR }}{n=16,530}$ & $\frac{\text { TAVR }}{n=4083}$ & $P$ value \\
\hline Postprocedure LOS, d, median (IQR) & $6(5-9)$ & $7(5-9)$ & $5(4-8)$ & $<.001$ \\
\hline ICU LOS, d, median (IQR) & $2(0-5)$ & $3(1-6)$ & $2(0-5)$ & $<.001$ \\
\hline 30-d mortality, n (\%) & $799(4)$ & $581(4)$ & $218(6)$ & $<.001$ \\
\hline $\begin{array}{c}\text { Propensity score-matched SAVR } \\
\text { and TAVR patients }\end{array}$ & $\mathrm{n}=6608$ & $\mathbf{n}=\mathbf{3 3 0 4}$ & $\mathbf{n}=3304$ & \\
\hline Postprocedure LOS, d, median (IQR) & $7(4-9)$ & $8(6-11)$ & $5(3-7)$ & $<.001$ \\
\hline ICU LOS, d, median (IQR) & $3(0-6)$ & $4(1-8)$ & $2(0-4)$ & $<.001$ \\
\hline 30-d mortality, n (\%) & $356(6)$ & $193(6)$ & $163(5)$ & .230 \\
\hline
\end{tabular}

$S A V R$, Surgical aortic valve replacement; TAVR, transcatheter aortic valve replacement; $L O S$, length of stay; $I Q R$, interquartile range; $I C U$, intensive care unit.

\section{Costs and Payments}

In 2012, Medicare paid \$1.02 billion for 20,613 AVRs (16,530 SAVR, 4083 TAVR), up from $\$ 901$ million for 18,391 isolated AVRs (17,043 SAVR, 1338 TAVR) in 2011. The rate of TAVR cases and the mean Medicare payment for isolated AVR increased in 2012 (Figure 1). Medicare paid $\$ 215,770,200$ nationally for TAVR in 2012, the first year of commercial implantation. Total perhospitalization costs for TAVR index hospitalizations in 2012 were higher than those for SAVR index hospitalizations (median, $\$ 50,700$ [IQR $\$ 40,200-\$ 65,700]$ for TAVR vs $\$ 39,400$ [IQR, $\$ 30,600-\$ 53,100]$ for SAVR; $P<.01$ ).

The median Medicare payment for TAVR index hospitalizations in 2012 was $\$ 50,100$ (IQR, \$37,500$\$ 65,700$ ), significantly higher than that for SAVR index hospitalizations $\quad(\$ 42,400 ; \quad I Q R, \quad \$ 32,800-\$ 56,300$; $P<.001)$. The contribution margin, defined as the median of the differences between the payment and estimated hospital cost, among AVR patients was negative for TAVR hospitalizations (-\$3200; representing a net negative contribution margin) and positive for SAVR hospitalizations (\$2700; representing a net positive contribution margin; $P<.01)$. More than one-half $(56 \% ; \mathrm{n}=11,443)$ of all TAVR or SAVR admissions resulted in net positive contribution margin, with a significantly greater percentage of SAVR admissions than TAVR admissions resulting in a positive contribution margin $(58 \%[\mathrm{n}=9642]$ vs $44 \%$ $[\mathrm{n}=1801] ; P<.01)$.

\section{Propensity Score-Matched TAVR and SAVR Patients}

Patient outcomes. Propensity score matching generated 3304 pairs of TAVR and SAVR patients. In the matched sample, TAVR patients remained more likely than SAVR patients to be white $(94 \%$ vs $92 \% ; P=.01)$, but there were no other significant differences between the TAVR and SAVR patients across age and common comorbidities (Table 3). Thirty-day mortality was not significantly different between the 2 groups in the propensity scorematched sample $(5 \%$ after TAVR, $6 \%$ after SAVR; $P=.23$ ). Postprocedure LOS remained significantly shorter

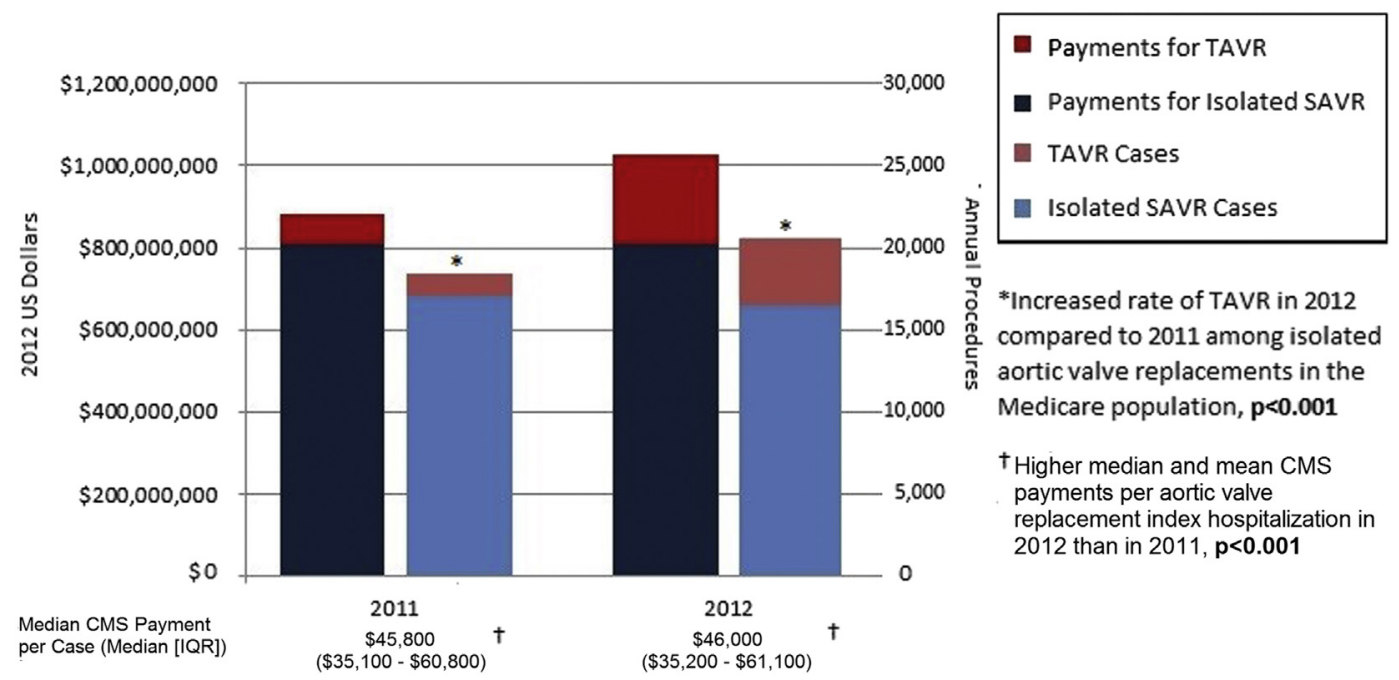

FIGURE 1. Annual CMS payments for TAVR and isolated SAVR in 2011 and 2012. CMS, Center for Medicare and Medicaid Services; TAVR, transcatheter aortic valve replacement; $S A V R$, surgical aortic valve replacement; $I Q R$, interquartile range. 
TABLE 3. Baseline characteristics of propensity score-matched SAVR and TAVR patients

\begin{tabular}{|c|c|c|c|c|}
\hline Characteristic & $\begin{array}{c}\text { All } \\
(\mathrm{n}=6608)\end{array}$ & $\begin{array}{c}\text { SAVR } \\
(n=3304)\end{array}$ & $\begin{array}{c}\text { TAVR } \\
(\mathbf{n}=\mathbf{3 3 0 4})\end{array}$ & $P$ value \\
\hline Age, $y$, mean \pm SD & $81.5 \pm 7.0$ & $81.4 \pm 7.4$ & $81.6 \pm 8.2$ & .089 \\
\hline Male sex & $3520(53)$ & $1786(54)$ & $1734(52)$ & .115 \\
\hline White race & $6162(93)$ & $3056(92)$ & $3106(94)$ & .013 \\
\hline Black race & $256(4)$ & $133(4)$ & $123(4)$ & .509 \\
\hline Congestive heart failure & $5771(87)$ & $2893(88)$ & $2878(87)$ & .415 \\
\hline Pulmonary circulation disorder & $2818(43)$ & $1414(43)$ & $1404(42)$ & .761 \\
\hline Peripheral vascular disease & $4737(72)$ & $2389(72)$ & $2348(71)$ & .121 \\
\hline Neurologic disorder & $913(14)$ & $461(14)$ & $452(14)$ & .723 \\
\hline Chronic obstructive pulmonary disease & $3319(50)$ & $1657(50)$ & $1662(50)$ & .872 \\
\hline Diabetes (complicated) & $1235(19)$ & $615(19)$ & $620(19)$ & .855 \\
\hline Renal failure & $2926(44)$ & $1473(45)$ & $1453(44)$ & .530 \\
\hline Liver disease & $228(3)$ & $111(3)$ & $117(4)$ & .683 \\
\hline Coagulopathy & $1243(19)$ & $650(20)$ & $593(18)$ & .071 \\
\hline Obesity & $1243(19)$ & $595(18)$ & $648(20)$ & .060 \\
\hline Weight loss & $548(8)$ & $262(8)$ & $286(9)$ & .258 \\
\hline Anemia & $3230(49)$ & $1614(49)$ & $1616(49)$ & .949 \\
\hline Depression & $888(13)$ & $432(13)$ & $456(14)$ & .339 \\
\hline Hypertension (complicated) & $6295(95)$ & $3135(95)$ & $3160(96)$ & .144 \\
\hline
\end{tabular}

Data are n (\%) unless noted otherwise. SAVR, Surgical aortic valve replacement; TAVR, transcatheter aortic valve replacement; $S D$, standard deviation.

for the TAVR patients (median, 5 days [IQR, 3-7 days] vs 8 days [IQR, 6-11 days]; $P<.01$ ), as did total ICU stay (median, 2 days [IQR, 2-4 days] vs 3 days [IQR, 1-6 days]; $P<.01$ ) (Table 2).

Costs. In the propensity score-matched sample, estimated hospital costs were significantly higher for TAVR compared with SAVR (median, $\$ 50,200$ [IQR, $\$ 39,800-\$ 64,300]$ vs $\$ 45,500$ [IQR, \$34,500-\$63,300]; $P<.01$ ) (Table 4). Compared with SAVR, index hospitalization costs for medical/surgical supplies and cardiology care were higher for
TAVR, but ICU charges, operating room charges, anesthesia charges, and blood administration charges were significantly lower for TAVR (Figure 2). (Note that physician fees are not included in our total hospital cost analysis.) Payments. Medicare payments were lower for TAVR hospitalizations compared with SAVR hospitalizations (median, \$49,500 [IQR, \$36,900-\$64,600] vs \$50,400 [IQR, $\$ 37,400-\$ 65,800] ; P<.01)$ in the propensity score-matched sample. The base DRG price amount was lower on average for TAVR hospitalizations (median,

TABLE 4. Procedural and financial outcomes in propensity score-matched SAVR and TAVR patients

\begin{tabular}{|c|c|c|c|}
\hline Outcome & $\operatorname{SAVR}(n=3304)$ & TAVR $(n=3304)$ & $P$ value \\
\hline \multicolumn{4}{|l|}{ Payments } \\
\hline Medicare payments, USD, median (IQR) & $50,400(37,400-65,800)$ & $49,500(36,900-64,600)$ & $<.01$ \\
\hline Base DRG price amount, USD, median (IQR) & $48,800(36,900-62,000)$ & $46,800(35,300-61,300)$ & $<.01$ \\
\hline Received DRG outlier payment, n (\%) & $541(16)$ & $705(21)$ & $<.01$ \\
\hline DRG outlier payments median, USD, (IQR) & $10,800(4600-25,600)$ & $7800(3600-14,700)$ & $<.01$ \\
\hline Received IME payment, n (\%) & $2246(74)$ & $2694(82)$ & $<.01$ \\
\hline IME payment amount median, USD, (IQR) & $2300(0-7400)$ & $4400(550-9600)$ & $<.01$ \\
\hline $\begin{array}{l}\text { Among hospitals that received DRG outlier } \\
\text { payments, USD, median (IQR)* }\end{array}$ & $4700(1500-9400)$ & $5900(2800-10,100)$ & $<.01$ \\
\hline Eligible for transfer penalty, $\mathrm{n}(\%)$ & $1251(38)$ & $2296(69)$ & $<.01$ \\
\hline \multicolumn{4}{|l|}{ Costs } \\
\hline Estimated hospital costs, USD, median (IQR) & $45,500(34,500-63,300)$ & $50,200(39,800-64,300)$ & $<.01$ \\
\hline Contribution margin, USD, median (IQR)* & 2390 ( -9000 to 13,300$)$ & $-3380(-14,300$ to 11,200$)$ & $<.01$ \\
\hline
\end{tabular}

$S A V R$, Surgical aortic valve replacement; TAVR, transcatheter aortic valve replacement; $U S D$, US dollar; $I Q R$, interquartile range; $D R G$, diagnosis-related group; IME, indirect medical education. *Defined as median of payments minus costs. 

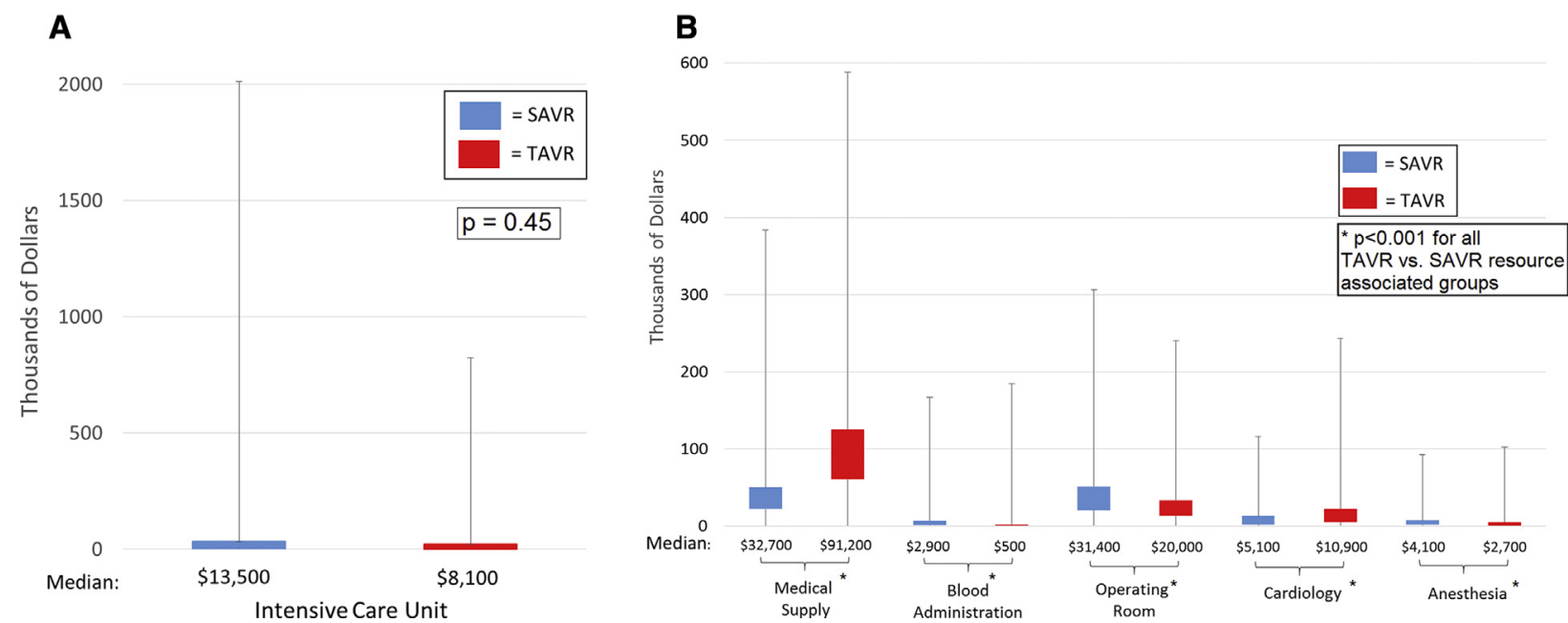

FIGURE 2. A, Median ICU costs for propensity-matched SAVR and TAVR patients. The lower quartile, median, and upper quartile represent the boxplot, and the minimum and maximum values are shown through the whisker extension on either side of the boxplot, indicating the variation in cost for TAVR and SAVR patients. B, Median resource-associated costs for propensity-matched SAVR and TAVR patients. The lower quartile, median, and upper quartile represent the boxplot, and the minimum and maximum values are shown through the whisker extension on either side of the boxplot, indicating the variation in cost for propensity-matched TAVR and SAVR patients. SAVR, Surgical aortic valve replacement; TAVR, transcatheter aortic valve replacement.

$\$ 46,800 \quad[\mathrm{IQR}, \quad \$ 35,300-\$ 61,300]$ vs $\$ 48,800 \quad[\mathrm{IQR}$, $\$ 36,900-\$ 62,000] ; P<.001)$. In the propensity scorematched sample, TAVR index hospitalizations were significantly more likely than SAVR hospitalizations to receive approved outlier payments above the base DRG amount approved for high-cost hospitalizations $(21 \%$ vs $16 \% ; P<.001)$, but the median outlier payments were lower for TAVR hospitalizations (median, \$7800 [IQR, $\$ 3600-\$ 14,700]$ vs $\$ 10,800$ [IQR, \$4600-\$25,600]; $P<.01)$. Compared with SAVR hospitalization payments, TAVR hospitalization payments were more likely to include additional funds for indirect medical education, and TAVR hospitals received higher average indirect medical education payments (Table 4).

Based on the difference between the billed DRG's national average LOS and the individual patient's LOS, TAVR admissions were much more likely to qualify for a post-acute care payment adjustment (ie, transfer penalty), which can potentially result in a DRG-specific down-weighting of reimbursement for admissions that are shorter relative to the national mean LOS. Among TAVR admissions, $69 \%$ qualified for DRG-specific down-weighting, compared with $38 \%$ of SAVR admissions $(P<.01)$ (Table 2). As in the entire cohort, the institutional contribution margin (ie, difference between payments and estimated hospital costs) in matched patients remained negative for TAVR hospitalizations ( $\$ 3380$, representing a net loss) and positive for SAVR hospitalizations ( $\$ 2390$, representing a net gain; $P<.01)$. A net financial gain, or positive contribution margin, occurred in $56 \%(\mathrm{n}=1862)$ of propensity score-matched SAVR admissions and $43 \%$ $(\mathrm{n}=1440)$ of propensity score-matched TAVR admissions
$(P<.01)$. For hospitals to experience no loss on TAVR hospitalizations, overall costs would need to decrease by $7 \%$, or isolated medical supply costs (related primarily to the TAVR valve) would need to decrease by $22 \%$.

Sensitivity analysis. Results from the sensitivity analysis using hierarchical multivariable logistic regression model to account for possible clustering of outcomes at certain centers did not show any difference in 30-day mortality between TAVR and SAVR (odds ratio, 0.88; 95\% confidence interval, 0.73-1.06; $P=.18$ ). An additional sensitivity analysis evaluating patients only at institutions performing TAVR found similar outcomes in terms of shorter ICU stays, similar mortality, higher costs, and lower payments for TAVR patients (Table E1). When the analysis was limited to propensity score-matched TF TAVR and SAVR patients, there was no longer a difference in median estimated cost $(\$ 49,200$ [IQR, $\$ 39,000-\$ 63,200]$ vs $\$ 44,800$ [IQR, $\$ 34,500-\$ 63,200] ; P=.37$ ), but other findings, including ICU LOS, 30-day mortality, payments, and contribution margin, were similar to those of the primary analysis (Table E2).

\section{Institutional Outcomes: TAVR Revenue and Volume}

A total of 1100 institutions were represented in the 2012 AVR sample, 207 of which performed at least 1 TAVR. Figure 3 shows the weighted contribution margin per patient for each institution based on the institution's 2012 volume of TAVR cases. Although the per-patient contribution margin varied widely among hospitals with similar case volumes, a cutoff emerged near 50 annual TAVR cases, at which point most hospitals achieved a net positive per-patient contribution margin. The average annual TAVR 


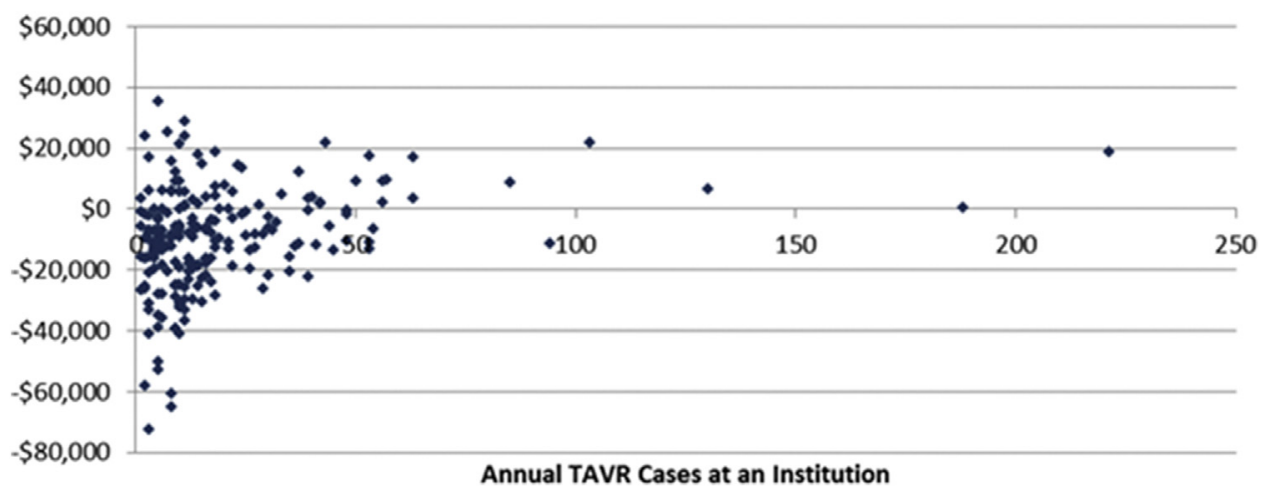

FIGURE 3. TAVR contribution margin per patient by institutional TAVR volume. TAVR, Transcatheter aortic valve replacement.

contribution margin weighted per patient was higher in hospitals that performed $\geq 50$ TAVRs $(\mathrm{n}=16$ hospitals; median, \$7761; IQR, $-\$ 2756$ to $\$ 13,396$ ) compared with hospitals that performed $<50$ TAVRs $(\mathrm{n}=191$; median, -\$9037; IQR, $-\$ 19,329$ to $-\$ 312 ; P<.01)$. This difference was driven primarily by higher Medicare payments at the hospitals performing $\geq 50$ TAVRs (median, \$56,600; IQR, $\$ 50,900-\$ 60,700)$ compared with the hospitals performing $<50$ TAVRs (median, \$45,100; IQR, \$36,900-\$58,800; $P=.01$ ), rather than periprocedural cost differences (median, $\$ 56,900 \quad[\mathrm{IQR}, \$ 44,500-\$ 61,800]$ vs $\$ 55,900$ [IQR, $\$ 47,400-\$ 66,000] ; P=.07)$.

\section{DISCUSSION}

TAVR is a new and expensive technology with economic implications in that have yet to be comprehensively evaluated in the United States. Any interpretation of the economic impact of TAVR should take into account the novelty of TAVR, the high-risk nature of the patient population, and the alternative therapeutic option of SAVR for some patients. This study describes the costs and payments associated with TAVR and SAVR in the United States from both the payer perspective and the provider perspective, and at the patient level for all Medicare beneficiaries and at the institutional level for individual institutions performing TAVR. Clinical trials continue to examine the safety and efficacy of TAVR in increasingly lower-risk AVR candidates and may further expand the TAVR-eligible population and increase the annual cost of TAVR to society beyond the $\$ 215$ million dollars in the first year of use.

We found that TAVR hospitalizations were more costly, or failed to generate revenue for hospitals, compared with SAVR hospitalizations in 2012. This was despite shorter ICU LOS and overall hospital LOS for TAVR patients compared with SAVR patients. Within a propensity score-matched sample of TAVR and SAVR patients, Medicare paid individual hospitals less on average for TAVR hospitalizations than for SAVR hospitalizations. TAVR hospitalizations represented a net loss of income for hospitals in the propensity score-matched sample and in the entire sample, whereas SAVR index hospitalizations resulted in a net positive contribution margin to hospitals in both samples. For TAVR to become financially neutral for hospitals, either Medicare and/or insurance companies must increase payments, hospitals must find a way to further reduce costs associated with TAVR, or the medical supply cost of TAVR, which is driven largely by the cost of the valve prosthesis, must decrease.

\section{Costs}

Our findings in this study are consistent with a model of estimated costs from the PARTNER trials and other studies. ${ }^{10,11}$ The higher cost for TAVR in the entire Medicare isolated AVR population may reflect the higherrisk status of TAVR patients, who overall represent a generally older and sicker cohort compared with patients undergoing SAVR. However, it is unlikely that all of the cost differences can be explained by patient risk factors. Even in the propensity score-matched cohort, TAVR hospitalization operating room, anesthesia, and blood administration costs were lower than those for SAVR in propensity score-matched patients, consistent with previous studies. ${ }^{20}$ Cardiology and medical/surgical supplies costs were higher for TAVR. The higher cost of surgical supplies can be explained in part by the more expensive TAVR valve systems, which are approximately $\$ 25,000$ more expensive than SAVR valves. ${ }^{9,21}$ These higher medical device costs are partially offset by shorter hospital and ICU stays for TAVR patients, 3 days shorter on average for the latter in the propensity score-matched sample. Considering that the sensitivity analysis of TF TAVR versus SAVR no longer showed a difference in cost, this may be a more significant finding in alternative access TAVR, but may be more relevant to future transcatheter technologies. The similar cost of TF TAVR and SAVR has important implications in terms of the increasing use of TF TAVR, which may make it more affordable to institutions. Conversely, the expanded use of 
TAVR into lower-risk patients may more closely approach the non-propensity score-matched sample in this study, which showed a greater cost difference in TAVR compared with SAVR.

\section{Payments}

Although Medicare payments were higher for TAVR than for SAVR in the entire Medicare sample, they were lower for TAVR than for SAVR in the propensity score-matched sample. The difference in the entire sample is most likely due to the higher risk status of TAVR patients compared with all SAVR patients, but the lower payments among propensity score-matched patients suggests that institutions performing TAVR face systematic barriers to recouping the costs of TAVR compared with similarly high-risk SAVR patients. TAVR index hospitalizations were billed at a lower base DRG price compared with SAVR and were more likely to be eligible for post-acute care payment adjustments (ie, transfer penalties that decrease the payment to hospitals, which are accounted for in the final Medicare payment amount), owing to the shorter LOS for TAVR patients. An advantage of using Medicare payments for this analysis is that the payment already accounts for and captures the transfer penalty, because it represents final action on the part of Medicare payments to the hospital.

Recently instituted changes in coding for US Medicare patients, including a change to the International Classification of Diseases, 10th Revision codes and new TAVR DRGs with higher relative financial weights and shorter LOS may have a significant impact on TAVR payments. Whether these changes have a positive financial impact on the $71 \%$ of institutions performing TAVR experiencing a net negative institutional contribution margin on these procedures remains to be seen. It is possible that in addition to changes made by the CMS to TAVR DRGs, reductions in the cost TAVR prostheses will be necessary for TAVR to achieve financial neutrality for hospitals. This may occur with the introduction of multiple new TAVR prostheses to the market and greater price competition among TAVR prosthetic manufacturers.

\section{Health Policy and Reimbursement Implications}

The costs and payments associated with TAVR are likely of particular interest to health policy makers and CMS for several reasons. First, TAVR is a new and disruptive technology for treating structural heart disease with high medical supply costs, but it is unlikely to be the last, with a number of other transcatheter aortic and mitral valve interventions as well ventricular assist devices currently in various phases of development, preapproval clinical trials, or postapproval surveillance. Second, the heart team model of cardiac surgeons and interventional cardiologists is a possible although not complete example of health care delivery through an Accountable Care Organization. Third, the FDA requirement that TAVR patients be entered into the Transcatheter Valve Registry is a good potential example of Merit-Based Incentive Payment System and Alternative Payment Model Incentive under the Physician Fee Schedule and Criteria for Physician-Focused Payment Models. Fourth, the push toward value-based care will likely focus on areas such as TAVR, where there are varying outcomes and costs between SAVR and TAVR.

To better inform both policy makers and providers, this study has focused on actual costs, payments and outcomes in propensity score-matched TAVR and SAVR cohorts. Although both interventions achieved excellent clinical results in relatively high-risk patient populations, the shorter ICU and hospital LOS, lower payments, and higher costs associated with TAVR represent a challenge to the CMS, insurance companies, physicians, hospitals, and industry to continue to meet the demand and deliver high-quality care in a way that balances the effort to both limit ongoing Medicare cost expenditures and encourage the development of better clinical interventions.

\section{Study Limitations}

Our analysis was based on administrative claims, which do not directly report institutional costs of care. Hospitalspecific cost-to-charge ratios have been previously used successfully to estimate actual cost of care from the total hospital charges reported on inpatient claims. ${ }^{17}$ In addition, the clinical information available on administrative claims is not as rich as data captured in cardiac surgery clinical trials or disease-specific registries, both of which may provide more detailed patient and procedural information. Claims data have been previously used successfully to propensity score match patients undergoing cardiac and other interventions. ${ }^{22,23}$ A cost analysis of TAVR compared with medical management was beyond the scope of this study. In addition, this study is limited by evaluating only the cost associated with TAVR or SAVR hospitalization and not evaluating longer-term costs, which may vary between groups and by technology. TAVR technology is rapidly evolving, and future studies are warranted to evaluate TAVR advances. Finally, although our propensity score match achieved a good balance of measured clinical characteristics, we cannot rule out the presence of unmeasured confounders in our analysis or confirm specific risk cohorts such as high-risk versus inoperable patients. In addition, it is possible that some TAVR patients might not have been candidates for SAVR owing primarily to comorbidities and consequently were predisposed to generating higher costs. The shorter hospital and ICU LOS of TAVR patients helps mitigate any concerns over higher patient-driven costs in TAVR. 


\section{CONCLUSIONS}

From the financial perspective of Medicare, TAVR, particularly TF TAVR, is an acceptable alternative to SAVR for high-risk SAVR candidates, with comparable outcomes and expenditures in this high-risk cohort. From an institutional provider perspective, however, SAVR generates revenue, whereas TAVR does not and instead incurs higher costs. These higher costs are driven primarily by the cost of medical supplies (the TAVR prostheses), with post-acute care adjustments also contributing. Overall reimbursement for TAVR appears to be misaligned with total hospital expenditures for prosthesis and periprocedural costs, particularly for alternative access TAVR.

\section{Conflict of Interest Statement}

J.E.B., H.C.H., S.A., W.Y.S., and N.D.D. are investigators in the Edwards Lifesciences PARTNER trial and Medtronic SURTAVI trial. H.C.H. also receives research funding from Edwards Lifesciences, Medtronic, Boston Scientific, and St. Jude Medical, and speaking honoraria (consultant) from Edwards LifeSciences. S.A. serves as an investigator for REPRISE and as a consultant for Medtronic. J.G. receives research funding from St. Jude Medical. All other authors have nothing to disclose with regard to commercial support.

\section{References}

1. Smith CR, Leon MB, Mack MJ, Miller DC, Moses JW, Svensson LG, et al. Transcatheter versus surgical aortic-valve replacement in high-risk patients. $N$ Engl J Med. 2011;364:2187-98.

2. Leon MB, Smith CR, Mack MJ, Makkar RR, Svensson LG, Kodali SK, et al. Transcatheter or surgical aortic-valve replacement in intermediate-risk patients. N Engl J Med. 2016;374:1609-20.

3. Leon MB, Smith CR, Mack M, Miller DC, Moses JW, Svensson LG, et al. Transcatheter aortic-valve implantation for aortic stenosis in patients who cannot undergo surgery. N Engl J Med. 2010;363:1597-607.

4. Mack MJ, Holmes DR Jr. Rational dispersion for the introduction of transcatheter valve therapy. JAMA. 2011;306:2149-50.

5. McCarthy FH, Groeneveld PW, Kobrin D, McDermott KM, Wirtalla C, Desai ND. Effect of clinical trial experience on transcatheter aortic valve replacement outcomes. Circ Cardiovasc Interv. 2015;8:e002234.

6. Reynolds MR, Magnuson EA, Lei Y, Wang K, Vilain K, Li H, et al. Cost-effectiveness of transcatheter aortic valve replacement compared with surgical aortic valve replacement in high-risk patients with severe aortic stenosis: results of the PARTNER (Placement of Aortic Transcatheter Valves) trial (Cohort A). J Am Coll Cardiol. 2012;60:2683-92.

7. Hancock-Howard RL, Feindel CM, Rodes-Cabau J, Webb JG, Thompson AK, Banz K. Cost effectiveness of transcatheter aortic valve replacement compared to medical management in inoperable patients with severe aortic stenosis:
Canadian analysis based on the PARTNER trial Cohort B findings. J Med Econ. 2013;16:566-74.

8. Simons CT, Cipriano LE, Shah RU, Garber AM, Owens DK, Hlatky MA. Transcatheter aortic valve replacement in nonsurgical candidates with severe, symptomatic aortic stenosis: a cost-effectiveness analysis. Circ Cardiovasc Qual Outcomes. 2013;6:419-28.

9. Gadey G, Reynolds MR. Cost-effectiveness considerations in transcatheter management of valvular heart disease. Can J Cardiol. 2014;30:1058-63.

10. Clark MA, Arnold SV, Duhay FG, Thompson AK, Keyes MJ, Svensson LG, et al. Five-year clinical and economic outcomes among patients with medically managed severe aortic stenosis: results from a Medicare claims analysis. Circ Cardiovasc Qual Outcomes. 2012;5:697-704.

11. Minutello RM, Wong SC, Swaminathan RV, Feldman DN, Kaple RK, Horn EM, et al. Costs and in-hospital outcomes of transcatheter aortic valve implantation versus surgical aortic valve replacement in commercial cases using a propensity score-matched model. Am J Cardiol. 2015;115:1443-7.

12. Pollak PM, Mack MJ, Holmes DR Jr. Quality, economics, and national guidelines for transcatheter aortic valve replacement. Prog Cardiovasc Dis. 2014;56:610-8.

13. Boothroyd LJ, Spaziano M, Guertin JR, Lambert LJ, Rodés-Cabau J, Noiseux N, et al. Transcatheter aortic valve implantation: recommendations for practice based on a multidisciplinary review including cost-effectiveness and ethical and organizational issues. Can J Cardiol. 2013;29:718-26.

14. Brennan JM, Holmes DR, Sherwood MW, Edwards FH, Carroll JD, Grover FL, et al. The association of transcatheter aortic valve replacement availability and hospital aortic valve replacement volume and mortality in the United States. Ann Thorac Surg. 2014;98:2016-22; discussion 2022.

15. United States Department of Labor, Bureau of Labor Statistics. CPI inflation calculator. Available at: http://www.bls.gov/data/inflation_calculator. htm. Accessed July 8, 2017.

16. Elixhauser A, Steiner C, Harris DR, Coffey RM. Comorbidity measures for use with administrative data. Med Care. 1998;36:8-27.

17. Chen LM, Jha AK, Guterman S, Ridgway AB, Orav EJ, Epstein AM. Hospital cost of care, quality of care, and readmission rates: penny wise and pound foolish? Arch Intern Med. 2010;170:340-6.

18. Lindenauer PK, Rothberg MB, Pekow PS, Kenwood C, Benjamin EM, Auerbach AD. Outcomes of care by hospitalists, general internists, and family physicians. N Engl J Med. 2007;357:2589-600.

19. Grzybowski M, Clements EA, Parsons L, Welch R, Tintinalli AT, Ross MA, et al. Mortality benefit of immediate revascularization of acute ST-segment elevation myocardial infarction in patients with contraindications to thrombolytic therapy: a propensity analysis. JAMA. 2003;290:1891-8.

20. Osnabrugge RL, Head SJ, Genders TS, Van Mieghem NM, De Jaegere PP, van der Boon RM, et al. Costs of transcatheter versus surgical aortic valve replacement in intermediate-risk patients. Ann Thorac Surg. 2012;94:1954-60.

21. Lynch AM. Re: Medicare program: hospital inpatient prospective payment systems for acute care hospitals and the long-term care hospital prospective payment system and fiscal year 2013 rates. June 25, 2012. Available at: https://www. advamed.org/sites/default/files/resource/507_2012_0625_advamed_ipps_fy_ 2013_comments.pdf. Accessed August 22, 2015.

22. Du DT, McKean S, Kelman JA, Laschinger J, Johnson C, Warnock R, et al. Early mortality after aortic valve replacement with mechanical prosthetic vs bioprosthetic valves among Medicare beneficiaries: a population-based cohort study. JAMA Intern Med. 2014;174:1788-95.

23. Schermerhorn ML, O'Malley AJ, Jhaveri A, Cotterill P, Pomposelli F, Landon BE. Endovascular vs. open repair of abdominal aortic aneurysms in the Medicare population. N Engl J Med. 2008;358:464-74.

Key Words: AVR, TAVR, SAVR, cost 
TABLE E1. Outcomes, costs, and payments for TAVR and SAVR patients at institutions performing TAVR

\begin{tabular}{|c|c|c|c|}
\hline Variable & $\operatorname{SAVR}(n=2826)$ & $\operatorname{TAVR}(\mathrm{n}=\mathbf{2 8 2 6})$ & $P$ value \\
\hline Postprocedure LOS, d, median (IQR) & $8(6-11)$ & $5(3-7)$ & $<.01$ \\
\hline ICU LOS, d, median (IQR) & $4(1-8)$ & $2(0-4)$ & $<.01$ \\
\hline 30-d mortality, n (\%) & $134(5)$ & $126(5)$ & .95 \\
\hline Medicare payments, USD, median (IQR) & $53,200(40,500-68,300)$ & $48,900(36,700-63,700)$ & $<.01$ \\
\hline Estimated hospital costs, USD, median (IQR) & $44,800(34,500-62,300)$ & $50,000(39,700-64,200)$ & $<.01$ \\
\hline Contribution margin, USD, median (IQR)* & $5800(-5300$ to 16,700$)$ & $-3600(-14,400$ to 10,600$)$ & $<.01$ \\
\hline
\end{tabular}

$S A V R$, Surgical aortic valve replacement; TAVR, transcatheter aortic valve replacement; $L O S$, length of stay; $I Q R$, interquartile range; $I C U$, intensive care unit; $U S D$, US dollar. *Defined as median of payments minus costs.

TABLE E2. Outcomes, costs, and payments for TF TAVR and SAVR patients

\begin{tabular}{|c|c|c|c|}
\hline Variable & TF $(n=2382)$ & $\operatorname{SAVR}(n=2826)$ & $P$ values \\
\hline Postprocedure LOS, d, median (IQR) & $5(3-7)$ & $8(6-11)$ & $<.01$ \\
\hline ICU LOS, d, median (IQR) & $2(0-4)$ & $4(2-8)$ & $<.01$ \\
\hline 30-d mortality, n (\%) & $97(4)$ & $134(5)$ & .34 \\
\hline Medicare payments, USD, median (IQR) & $47,500(36,000-62,200)$ & $53,100(40,500-68,300)$ & $<.01$ \\
\hline Eligible for transfer penalty, $\mathrm{n}(\%)$ & $1761(74)$ & $1092(39)$ & $<.01$ \\
\hline Estimated hospital costs, USD, median (IQR) & $49,200(39,000-63,200)$ & $44,800(34,500-62,300)$ & .37 \\
\hline Contribution margin, USD, median (IQR)* & $-3500(-14,100$ to 10,800$)$ & $5800(-5300$ to 16,700$)$ & $<.01$ \\
\hline
\end{tabular}

$T F$, Transfemoral; SAVR, surgical aortic valve replacement; $L O S$, length of stay; IQR, interquartile range; ICU, intensive care unit; USD, US dollar. *Defined as median of payments minus costs. 